IFT-UAM/CSIC-05-46

\title{
Reconstructing the two right-handed neutrino model
}

\author{
Alejandro Ibarra ${ }^{\dagger}$ \\ Instituto de Física Teórica, CSIC/UAM, C-XVI \\ Universidad Autónoma de Madrid, \\ Cantoblanco, 28049 Madrid, Spain.
}

October 28, 2018

\begin{abstract}
In this paper we propose a low-energy parametrization of the two right-handed neutrino model, and discuss the prospects to determine experimentally these parameters in supersymmetric scenarios. In addition, we present exact formulas to reconstruct the high-energy leptonic superpotential in terms of the low-energy observables. We also discuss limits of the three right-handed neutrino model where this procedure applies.
\end{abstract}

\footnotetext{
${ }^{\dagger}$ E-mail: alejandro.ibarra@cern.ch
} 


\section{Introduction}

The observed neutrino masses can be naturally accounted for by adding to the Standard Model Lagrangian three heavy singlets, usually identified with the right-handed neutrinos. This framework is denoted as the type I see-saw mechanism, or simply the see-saw mechanism [1]. Furthermore, this framework can also accommodate, although not explain, the large atmospheric and solar mixing angles, as well as the small 13 element in the leptonic mixing matrix.

Although the see-saw mechanism describes qualitatively well the observations, it lacks predictive power. The reason is that the leptonic Lagrangian is defined at high energies by 21 parameters, whereas experimentally we can only measure at most 12 parameters. The 9 remaining parameters are lost in the decoupling process and can be arbitrarily chosen, without leaving any other trace at low energies. ${ }^{1}$

In the supersymmetric version of the see-saw mechanism the neutrino Yukawa coupling affects the renormalization group evolution of the slepton parameters above the decoupling scale [3], and thus leave an imprint that could be disentangled using low energy experiments (after making some assumptions on the structure of the slepton parameters at the cut-off scale). For instance, phenomena such as rare decays [4,5] electric dipole moments [7, or mass splittings among the different generations of charged sleptons or sneutrinos [8] provide additional information about the see-saw parameters.

This information is encoded in a $3 \times 3$ hermitian matrix, $P=\mathbf{Y}_{\nu}^{\dagger} \mathbf{Y}_{\nu}$, that depends on nine parameters (six moduli and three phases). It can be shown that these nine parameters are precisely the complementary information needed to reconstruct the high energy Lagrangian [910]. The positive consequence of this observation is that the see-saw mechanism can be parametrized just in terms of low energy observables; the negative consequence is that for any set of low energy lepton and slepton parameters, there is a high energy theory with three right-handed neutrinos that can accommodate it, and in consequence, the see-saw model cannot be disproved.

In this parametrization, the correspondence between high energy and low energy parameters is one to one. Therefore, any additional hypothesis on the high-energy see-saw parameters would lead to predictions on the low energy parameters. Some well

\footnotetext{
${ }^{1}$ There is additional information encoded in dimension six operators, but unfortunately they are too suppressed to be observed experimentally [2].
} 
motivated assumptions have been proposed in the literature, such as texture zeros, symmetric matrices or the two right-handed neutrino model, and their consequences studied in a number of papers. In this work we will concentrate on the latter possibility [1112]. The motivation is the following. Leaving aside the LSND anomaly, experiments have measured two mass splittings, indicating that at least two new scales have to be introduced. The two new scales could correspond to the masses of two right handed neutrinos, being the third one not necessary to reproduce the oscillation experiments. Therefore, a model with just two right handed neutrinos could explain all the observations, but it depends on less parameters.

The two right-handed neutrino model has some interesting features. Namely, the two right-handed neutrino model predicts a hierarchical spectrum for the light neutrinos, being the lightest strictly massless. Furthermore, since there are only two non-vanishing masses, there is only one Majorana phase, corresponding to the phase difference between these two eigenvalues. Analogously to the model with three righthanded neutrinos, there are three mixing angles and one Dirac phase.

Since the number of parameters involved is smaller, we expect predictions not only for the neutrino mass matrix, but also for the low-energy slepton mass matrix (under some assumptions about the structure of this matrix at high energy), as well as a simpler reconstruction procedure of the high energy parameters. In section 2 we will show that in the two right-handed neutrino model some relations arise among different elements in the slepton mass matrix. We will also present a possible parametrization of this model just in terms of low energy observables. In Section 3 we will discuss the prospects to determine experimentally these parameters, and accordingly the feasibility of the reconstruction of the high-energy parameters. In section 4 we will propose an exact reconstruction procedure, and illustrate it for a particular possibility of the low energy parameters. In section 5 we will analyze different limits of the three right handed neutrino model that can be well described in practice by a two right-handed neutrino model, and where the reconstruction procedure proposed in this paper applies. Finally, in Section 6 we will present our conclusions. We will also present an appendix with a more elaborated reconstruction procedure. 


\section{Parametrizations of the two right-handed neu- trino model}

In the appropriate basis, the two right-handed neutrino (2RHN) model is defined at high energies by a $2 \times 3$ Yukawa matrix and two right-handed neutrino masses, $M_{1}$ and $M_{2}$. This amounts to eight moduli and three phases. On the other hand, at low energies the neutrino mass matrix is defined by five moduli (two masses and three mixing angles) plus two phases (the Dirac phase and the Majorana phase). Therefore, the number of unknown parameters is reduced to three moduli and one phase.

Using the parametrization presented in [5], the neutrino Yukawa coupling can be expressed as:

$$
\mathbf{Y}_{\nu}=D_{\sqrt{\mathbf{M}_{\nu}}} R D_{\sqrt{m}} U^{\dagger} /\left\langle H_{u}^{0}\right\rangle
$$

where $D_{\sqrt{\mathbf{M}_{\nu}}}=\operatorname{diag}\left(\sqrt{M_{1}}, \sqrt{M_{2}}\right)$ is the diagonal matrix of the square roots of the right-handed masses, $D_{\sqrt{m}}=\operatorname{diag}\left(0, \sqrt{m_{2}}, \sqrt{m_{3}}\right)$ is the diagonal matrix of the squared roots of the physical masses of the light neutrinos, $\left\langle H_{u}^{0}\right\rangle$ is the vacuum expectation value of the neutral component of the up-type Higgs doublet, $U$ is the leptonic mixing matrix [13], and $R$ is a $2 \times 3$ complex matrix, which parametrizes the information that is lost in the decoupling of the right-handed neutrinos. It is possible to prove that $R$ has the following structure [1415]

$$
R=\left(\begin{array}{ccc}
0 & \cos z & \xi \sin z \\
0 & -\sin z & \xi \cos z
\end{array}\right)
$$

where $z$ is a complex parameter and $\xi= \pm 1$ is a parameter that accounts for a discrete indeterminacy in the Yukawa coupling.

Notice that we have included all the low energy phases in the definition of the matrix $U$, i.e. we have written the leptonic mixing matrix in the form $U=V \operatorname{diag}\left(1, e^{-i \phi / 2}, 1\right)$, where $\phi$ is the Majorana phase and $V$ has the form of the CKM matrix:

$$
V=\left(\begin{array}{ccc}
c_{13} c_{12} & c_{13} s_{12} & s_{13} e^{-i \delta} \\
-c_{23} s_{12}-s_{23} s_{13} c_{12} e^{i \delta} & c_{23} c_{12}-s_{23} s_{13} s_{12} e^{i \delta} & s_{23} c_{13} \\
s_{23} s_{12}-c_{23} s_{13} c_{12} e^{i \delta} & -s_{23} c_{12}-c_{23} s_{13} s_{12} e^{i \delta} & c_{23} c_{13}
\end{array}\right),
$$

so that the neutrino mass matrix is $\mathcal{M}=U^{*} \operatorname{diag}\left(0, m_{2}, m_{3}\right) U^{\dagger}$. It is straightforward to check that eq.(II) indeed satisfies $\mathcal{M}=\mathbf{Y}_{\nu}^{T} \operatorname{diag}\left(M_{1}^{-1}, M_{2}^{-1}\right) \mathbf{Y}_{\nu}\left\langle H_{u}^{0}\right\rangle^{2}$.

The Yukawa coupling affects the renormalization group equation of the slepton parameters through the combination $P=\mathbf{Y}_{\nu}^{\dagger} \mathbf{Y}_{\nu}$, that depends in general on six moduli 
and three phases. Since the Yukawa coupling depends in the 2RHN model on only three unknown moduli and one phase, so does $P$, and consequently it is possible to obtain predictions on the moduli of three $P$-matrix elements and the phases of two $P$-matrix elements. Namely, from eq.(11) one obtains that:

$$
U^{\dagger} P U=U^{\dagger} \mathbf{Y}_{\nu}^{\dagger} \mathbf{Y}_{\nu} U=D_{\sqrt{m}} R^{\dagger} D_{\mathbf{M}_{\nu}} R D_{\sqrt{m}} /\left\langle H^{0}\right\rangle^{2}
$$

Since $m_{1}=0$, it follows that $\left(U^{\dagger} P U\right)_{1 i}=0$, for $i=1,2,3$, leading to three relations among the elements in $P$. For instance, one could derive the diagonal elements in $P$ in terms of the off-diagonal elements:

$$
\begin{aligned}
& P_{11}=-\frac{P_{12}^{*} U_{21}^{*}+P_{13}^{*} U_{31}^{*}}{U_{11}^{*}}, \\
& P_{22}=-\frac{P_{12} U_{11}^{*}+P_{23}^{*} U_{31}^{*}}{U_{21}^{*}}, \\
& P_{33}=-\frac{P_{13} U_{11}^{*}+P_{23} U_{21}^{*}}{U_{31}^{*}} .
\end{aligned}
$$

The observation of these correlations would be non-trivial tests of the $2 \mathrm{RHN}$ model.

The relations for the phases arise from the hermicity of $P$, since the diagonal elements in $P$ have to be real. Taking as the independent phase the argument of $P_{12}$, one can derive from eq.(5) the arguments of the remaining elements:

$$
\begin{aligned}
e^{i \arg P_{13}} & =\frac{-i \operatorname{Im}\left(P_{12} U_{21} U_{11}^{*}\right) \pm \sqrt{\left|P_{13}\right|^{2}\left|U_{11}\right|^{2}\left|U_{31}\right|^{2}-\left[\operatorname{Im}\left(P_{12} U_{21} U_{11}^{*}\right)\right]^{2}}}{\left|P_{13}\right| U_{31} U_{11}^{*}} \\
e^{i \arg P_{23}} & =\frac{i \operatorname{Im}\left(P_{12} U_{21} U_{11}^{*}\right) \pm \sqrt{\left|P_{23}\right|^{2}\left|U_{21}\right|^{2}\left|U_{31}\right|^{2}-\left[\operatorname{Im}\left(P_{12} U_{21} U_{11}^{*}\right)\right]^{2}}}{\left|P_{23}\right| U_{31} U_{21}^{*}}
\end{aligned}
$$

where the \pm sign has to be chosen so that the eigenvalues of $P$ are positive. It is important to remark that the hermicity of $P$ is not guaranteed for any value of $P_{12}$, $\left|P_{13}\right|,\left|P_{23}\right|$; only some particular ranges for the parameters are allowed, corresponding to the values for which the arguments of the square roots in eq.(6) are positive.

We conclude then that the $P$-matrix parameters $P_{12},\left|P_{13}\right|$ and $\left|P_{23}\right|$ can be regarded as independent and can be used as an alternative parametrization of the 2 RHN model. Together with the five moduli and the two phases of the neutrino mass matrix, sum up to the eight moduli and the three phases necessary to reconstruct the high-energy Lagrangian of the $2 \mathrm{RHN}$ model. 


\section{Can we reconstruct (realistically) the complete theory from low energy observables?}

An interesting feature of the $2 \mathrm{RHN}$ model is that it could be feasible to reconstruct the neutrino mass matrix. This model predicts that one of the neutrino masses vanishes, and in consequence the spectrum is necessarily hierarchical. In this scenario, the masses would have been already determined by present oscillation experiments: $m_{2}=\sqrt{\Delta m_{21}^{2}}$ and $m_{3}=\sqrt{\Delta m_{31}^{2}}$, where $\Delta m_{21}^{2}=(7.1-8.9) \times 10^{-5} \mathrm{eV}$ and $\Delta m_{31}^{2}=(1.4-3.3) \times 10^{-3} \mathrm{eV}$ are the $3 \sigma$ ranges for the solar and atmospheric mass splittings obtained from the combined analysis of global data [16]. In the next few years, the measurements of the mass splittings are expected to improve. To be specific, the error in the measurement of the atmospheric mass splitting, $\Delta m_{31}^{2}$, is expected to be reduced by experiments using the CERN to Gran Sasso neutrino beam (ICARUS [17] and OPERA [18]), MINOS [19], $\mathrm{NO} \nu \mathrm{A}[20$ ] and particularly T2K [21], that will probably reduce the present error by one order of magnitude. There are also proposals to reduce the error in the measurement of the solar mass splitting, $\Delta m_{21}^{2}$. Namely, if the SuperK detector was loaded with gadolinium, it would be possible to reduce the error by a factor of six [23].

Concerning the mixing angles, two of them have been determined to a good accuracy by present experiments: $\sin ^{2} \theta_{12}=0.24-0.40$ and $\sin ^{2} \theta_{23}=0.34-0.68$ at the $3 \sigma$ level [16]. The T2K experiment will probably reduce the error in $\sin ^{2} \theta_{23}$ by a factor of two. On the other hand, the error in $\sin ^{2} \theta_{12}$ will not be substantially reduced in the near future, although an experiment similar to KamLAND but with a baseline slightly shorter, $L \sim 60 \mathrm{~km}$, could reduce the error by a factor of four [24].

The angle $\theta_{13}$ has not been detected yet but the global analysis sets the upper bound $\sin ^{2} \theta_{13}<0.046$, also at $3 \sigma$ 16. Ongoing experiments such as MINOS, ICARUS or OPERA could improve the present limit by a factor of two, while future experiments as D-Chooz 22 by a factor of four, and $\mathrm{T} 2 \mathrm{~K}$ or $\mathrm{NO} \nu \mathrm{A}$ by a factor of ten. If $\theta_{13}$ is large, the combined analysis of the experiments could provide some information about the Dirac phase $\delta$. However, the detailed analysis of CP violation in the neutrino sector will require superbeams. For instance, improving the proton intensity at JHF to 4MW and using the proposed Hyper-Kamiokande detector, it could be possible to reach a sensitivity below $3 \times 10^{-4}$ for $\sin ^{2} \theta_{13}$ and around $10^{\circ}-20^{\circ}$ for $\delta$ [21].

Thus we find that there are good prospects to determine the masses, mixing angles 
and $\delta$ to the percent level in the next 10-20 years [25]. However, the measurement of the Majorana phase is still very challenging. Although it could in principle be measured in experiments of neutrinoless double beta decay, the sensitivity of the projected experiments is still far from observing this process in the case of a hierarchical spectrum of neutrinos [26].

Concerning the matrix $P$, there are also very good prospects to determine the relevant parameters: $P_{12},\left|P_{13}\right|$ and $\left|P_{23}\right|$. With experiments of rare decays, it would be possible to determine the moduli of the off-diagonal elements of $P$. The present bounds on the rates for these decays are $B R(\mu \rightarrow e \gamma) \lesssim 1.2 \times 10^{-11}$ [27], $B R(\tau \rightarrow$ $\mu \gamma)<3.1 \times 10^{-7}$ [28] (Belle) or $<6.8 \times 10^{-8}$ [29] (BaBar), and $B R(\tau \rightarrow e \gamma)<3.9 \times 10^{-7}$ 30], that translate into $\left|P_{12}\right|<3 \times 10^{-4},\left|P_{23}\right|<0.09$ and $\left|P_{13}\right|<0.09$, for $\tan \beta=10$ and typical slepton masses of $\sim 400 \mathrm{GeV}$ [31].

In the next few years, the sensitivity of the experiments to these processes is also expected to improve. The MEG experiment expects to reach a sensitivity of $B R(\mu \rightarrow$ $e \gamma) \lesssim 10^{-13}$ [32], that would translate into $\left|P_{12}\right| \lesssim 3 \times 10^{-5}$. Although presently the most stringent constraints on $\left|P_{12}\right|$ arise from the process $\mu \rightarrow e \gamma$, this role could be played in the future by experiments on the coherent conversion of muons into electrons in nuclei. ${ }^{2}$ The current experimental bounds are $R\left(\mu^{-} \mathrm{Ti} \rightarrow e^{-} \mathrm{Ti}\right)<6.1 \times 10^{-13}$ 33] and $R\left(\mu^{-} \mathrm{Au} \rightarrow e^{-} \mathrm{Au}\right)<5 \times 10^{-13}$ [34], and are expected to be improved by the PRISM/PRIME experiment, aiming to a sensitivity of $10^{-18}$ [35], or the CERN neutrino factory, aiming to $10^{-19}$ [36].

On the other hand, B-factories are also $\tau$-factories, and constitute splendid opportunities to search for rare $\tau$ decays. Future super B-factories could produce of the order of $10^{10} \tau$ pairs at a luminosity of $10 a b^{-1}$, allowing to probe branching ratios for the rare $\tau$ decays down to the level of $10^{-8}-10^{-9}$ [37.

The only phase that appears in $P$ could be determined from measurements of the electric dipole moment of the electron, whose present bound is $d_{e}<1.6 \times 10^{-27} \mathrm{e} \mathrm{cm}$ 38. In order to disentangle the contribution from the neutrino Yukawa couplings it would be necessary to determine the phases in the neutralino or the chargino sector from other experiments, most probably the LHC or the ILC. The prospects to improve the sensitivity of the experiments to detect electron dipole moments are also very

\footnotetext{
${ }^{2}$ When the photon penguin diagram dominates the contribution in the $\mu-e$ conversion, the conversion rate is approximately three orders of magnitude smaller than the branching ratio for $\mu \rightarrow e \gamma$.
} 
encouraging. By using the metastable $a(1)\left[{ }^{3} \Sigma^{*}\right]$ state of $\mathrm{PbO}$ it could be possible to improve the sensitivity of the experiments to $d_{e} \lesssim 10^{-29} \mathrm{e} \mathrm{cm}[39$, or even to $d_{e} \lesssim 10^{-31}$ e $\mathrm{cm}$ in a few years [40]. In the longer term, it could be possible to improve the sensitivity to $d_{e} \lesssim 10^{-35}$ e cm using solid state techniques [41. Incidentally, it has been argued that the effect of the phases in the neutrino Yukawa coupling would not be observed in the electron dipole moment before this sensitivity is reached 43 .

In summary, the prospects to detect or further constrain the low-energy parameters of the 2 RHN model are very encouraging, and accordingly the prospects to reconstruct the high energy theory from experiments. It is remarkable that most of the problems encountered when determining the low energy parameters of the 3RHN model disappear in the 2RHN model. Namely, in the 3RHN model there are two Majorana phases, and despite one combination of them could be measured in experiments of neutrinoless double beta decay, there is no proposed experimental set-up to measure the second combination. Furthermore, in the matrix $P$ there are more independent parameters in the $3 \mathrm{RHN}$ model than in the $2 \mathrm{RHN}$ model, and the prospects to measure them are not so encouraging. In particular, the diagonal elements in $P$ would be quite hard to measure, although it could be possible to measure the largest mass difference among the sleptons or the sneutrinos in colliders [8]. On the other hand, the measurement of the smallest mass difference seems to be far out of the reach of the proposed future experiments. Concerning the electric dipole moments, there are some prospects to improve the present bound on the muon electric dipole moment, $d_{\mu}<7 \times 10^{-19} \mathrm{e} \mathrm{cm}$ [44], to $d_{\mu} \lesssim 10^{-24} \mathrm{e} \mathrm{cm} \mathrm{using} \mathrm{the} \mathrm{muon} \mathrm{ring} \mathrm{at} \mathrm{BNL} \mathrm{[45]} \mathrm{or} \mathrm{even} \mathrm{to} d_{\mu} \lesssim 10^{-26} \mathrm{e} \mathrm{cm}$ at the neutrino factory 36 . On the other hand, the present bounds on the $\tau$ electric dipole moment are very loose, $-2.2<\operatorname{Re}\left(d_{\tau}\right)<4.5\left(\times 10^{-17}\right)$ e $\mathrm{cm}[46$, and there are no prospects to improve them substantially in the near future.

It was pointed out in 474815 , that the phase of $z$ is the only phase that plays a role in the mechanism of leptogenesis [49]. Therefore, the see-saw mechanism could be parametrized in terms of the leptogenesis phase instead of the phase that induces electric dipole moments. Nevertheless, the leptogenesis mechanism depends on assumptions that are harder to test than the assumptions needed to disentangle the phase in $P$ from electric dipole moments, and thus this possibility does not seem to be very practical. In any case, we find very remarkable the close relation between electric dipole moments and leptogenesis in the $2 \mathrm{RHN}$ model. 


\section{The reconstruction procedure}

In Section 2 we have discussed that the complete Lagrangian can be written in terms of the five moduli and two phases of the neutrino mass matrix, and the three independent moduli and two phases of the matrix $P$, that is involved in the radiative corrections of the slepton parameters. In this section we will derive exact formulas for the high energy parameters in terms of these low energy parameters [50]

To this end, we will use the parametrization of the Yukawa couplings in eq.(II), so that all our ignorance of the high energy theory is encoded in the right-handed neutrino masses, $M_{1}$ and $M_{2}$, and the complex parameter in the matrix $R, z$. Let us define the hermitian matrix $Q \equiv U^{\dagger} P U$, that depends exclusively on parameters that in principle could be measured in low energy experiments. The first row and column vanish and yield the relations among the $P$-matrix elements already presented in eq.(15). On the other hand, the remaining elements $Q_{22}, Q_{23}, Q_{33}$, can be written in terms of the highenergy parameters $M_{1}, M_{2}$ and $z$. Therefore, one can invert the equations to derive exact expressions for the high-energy parameters in terms of the low energy parameters in $Q$. These expressions are:

$$
\begin{aligned}
M_{1} & =\frac{1}{2}\left[\sqrt{\left(\frac{Q_{33}}{m_{3}}+\frac{Q_{22}}{m_{2}}\right)^{2}+\frac{\left(Q_{23}-Q_{23}^{*}\right)^{2}}{m_{2} m_{3}}}-\sqrt{\left(\frac{Q_{33}}{m_{3}}-\frac{Q_{22}}{m_{2}}\right)^{2}+\frac{\left(Q_{23}+Q_{23}^{*}\right)^{2}}{m_{2} m_{3}}}\right]\left\langle H_{u}^{0}\right\rangle^{2}, \\
M_{2} & =\frac{1}{2}\left[\sqrt{\left(\frac{Q_{33}}{m_{3}}+\frac{Q_{22}}{m_{2}}\right)^{2}+\frac{\left(Q_{23}-Q_{23}^{*}\right)^{2}}{m_{2} m_{3}}}+\sqrt{\left(\frac{Q_{33}}{m_{3}}-\frac{Q_{22}}{m_{2}}\right)^{2}+\frac{\left(Q_{23}+Q_{23}^{*}\right)^{2}}{m_{2} m_{3}}}\right]\left\langle H_{u}^{0}\right\rangle^{2}, \\
\cos 2 z & =\left(\frac{Q_{33}^{2}}{m_{3}^{2}}-\frac{Q_{22}^{2}}{m_{2}^{2}}+\frac{\left(Q_{23}+Q_{23}^{*}\right)\left(Q_{23}-Q_{23}^{*}\right)}{m_{2} m_{3}}\right) \frac{\left\langle H_{u}^{0}\right\rangle^{4}}{M_{2}^{2}-M_{1}^{2}} .
\end{aligned}
$$

To complete the reconstruction procedure, the Yukawa coupling would be derived from these parameters using eq.(11) and where the discrete parameter $\xi$ in eq.(2) is determined by:

$$
\xi=\frac{\sqrt{m_{2} m_{3}}}{Q_{23}\left\langle H_{u}^{0}\right\rangle^{2}}\left(M_{1} \sin z \cos z^{*}-M_{2} \cos z \sin z^{*}\right) .
$$

In the case that all the parameters are real, the expressions greatly simplify:

$$
\begin{aligned}
& M_{1}=\frac{1}{2}\left[\frac{Q_{33}}{m_{3}}+\frac{Q_{22}}{m_{2}}-\sqrt{\left(\frac{Q_{33}}{m_{3}}-\frac{Q_{22}}{m_{2}}\right)^{2}+\frac{4 Q_{23}^{2}}{m_{2} m_{3}}}\right]\left\langle H_{u}^{0}\right\rangle^{2}, \\
& M_{2}=\frac{1}{2}\left[\frac{Q_{33}}{m_{3}}+\frac{Q_{22}}{m_{2}}+\sqrt{\left(\frac{Q_{33}}{m_{3}}-\frac{Q_{22}}{m_{2}}\right)^{2}+\frac{4 Q_{23}^{2}}{m_{2} m_{3}}}\right]\left\langle H_{u}^{0}\right\rangle^{2},
\end{aligned}
$$




$$
\cos 2 z=\left(\frac{Q_{33}^{2}}{m_{3}^{2}}-\frac{Q_{22}^{2}}{m_{2}^{2}}\right) \frac{\left\langle H_{u}^{0}\right\rangle^{4}}{M_{2}^{2}-M_{1}^{2}} .
$$

We would like to illustrate now the reconstruction procedure for an interesting possibility for the matrix $P$. In the previous section we argued that among all the elements in $P$, the ones with better prospects to be constrained or measured were the off-diagonal ones. Therefore, it will prove convenient from the phenomenological point of view to use $P_{12},\left|P_{13}\right|$ and $\left|P_{23}\right|$, together with the neutrino mass matrix, to parametrize the $2 \mathrm{RHN}$ model. Furthermore, the stringent constraints on the process $\mu \rightarrow$ e $\gamma$ suggests us to take the limit $\left|P_{12}\right| \ll\left|P_{13}\right|,\left|P_{23}\right| .^{3}$ This limit yields a very constrained structure for the $P$-matrix,

$$
P \simeq\left(\begin{array}{ccc}
-P_{13}^{*} \frac{U_{31}^{*}}{U_{11}^{*}} & 0 & P_{13} \\
0 & -P_{23}^{*} \frac{U_{31}^{*}}{U_{21}^{*}} & P_{23} \\
P_{13}^{*} & P_{23}^{*} & -P_{13} \frac{U_{11}^{*}}{U_{31}^{*}}-P_{23} \frac{U_{21}^{*}}{U_{31}^{*}}
\end{array}\right)
$$

that in turn would imply predictions for the mass splittings among the sleptons in terms of the branching ratios for the processes $\tau \rightarrow \mu \gamma$ and $\tau \rightarrow e \gamma$. Note also that the phases in the matrix $P$ are fixed in terms of the phases in the leptonic mixing matrix. Namely, to make the diagonal elements of the matrix $P$ real, as required by hermicity, one has to require

$$
\begin{aligned}
& \arg P_{13} \simeq \arg U_{11}-\arg U_{31}+\pi, \\
& \arg P_{23} \simeq \arg U_{21}-\arg U_{31}+\pi .
\end{aligned}
$$

We have also resolved the \pm ambiguity in the expressions for the arguments of $P_{13}$ and $P_{23}$ in eq.(6) by applying the Sylvester criterion, in order to yield positive eigenvalues in $P$. Note that the Majorana phase, $\phi$, will not appear in these expressions, and the only phase that will appear is the Dirac phase, suppressed by the small value of $\theta_{13}$. To be precise, if we substitute $\theta_{23} \simeq \pi / 4, \theta_{12} \simeq \pi / 6$ and we take into account that $\theta_{13}$ is small, we obtain

$$
\begin{aligned}
& P_{13} \simeq-\left|P_{13}\right|\left(1+i \sqrt{3} \sin \delta \sin \theta_{13}\right), \\
& P_{23} \simeq\left|P_{23}\right|\left(1+2 i \sqrt{3} \sin \delta \sin \theta_{13}\right) .
\end{aligned}
$$

\footnotetext{
${ }^{3}$ It is important to stress that there is no solid experimental evidence supporting this possibility. The only reason why the bound on $\mu \rightarrow e \gamma$ is stronger than the bounds on $\tau \rightarrow(\mu, e) \gamma$ is that presently the muon sources are more intense than the tau sources. Other possibilities are a priori equally plausible from the phenomenological point of view, despite there is a theoretical prejudice in favor of $\left|P_{12}\right| \ll\left|P_{13}\right|,\left|P_{23}\right|$. The analysis for other scenarios will be presented elsewhere.
} 
Therefore, in the limit where $\left|P_{12}\right| \ll\left|P_{13}\right|,\left|P_{23}\right|$, all the elements in $P$ are expected to be real to a good approximation, and accordingly the contribution to the electric dipole moments from the slepton parameters is expected to be very small. Note also that requiring positive eigenvalues for $P$ requires that $P_{13}$ is negative and $P_{23}$ positive.

The high-energy parameters can be easily reconstructed from the general formulas in eq.(17), where the relevant elements in the matrix $Q$ read:

$$
\begin{aligned}
& Q_{22} \simeq\left|P_{13}\right| \frac{\left|U_{23}\right|^{2}}{\left|U_{11}\right|\left|U_{31}\right|}+\left|P_{23}\right| \frac{\left|U_{13}\right|^{2}}{\left|U_{21}\right|\left|U_{31}\right|}, \\
& Q_{33} \simeq\left|P_{13}\right| \frac{\left|U_{22}\right|^{2}}{\left|U_{11}\right|\left|U_{31}\right|}+\left|P_{23}\right| \frac{\left|U_{12}\right|^{2}}{\left|U_{21}\right|\left|U_{31}\right|}, \\
& Q_{23} \simeq-\left|P_{13}\right| \frac{U_{22}^{*} U_{23}}{\left|U_{11}\right|\left|U_{31}\right|}-\left|P_{23}\right| \frac{U_{12}^{*} U_{13}}{\left|U_{21}\right|\left|U_{31}\right|} .
\end{aligned}
$$

To show the analytical results, we will limit ourselves to the case where $\theta_{23} \simeq \pi / 4$, $\theta_{12} \simeq \pi / 6$, and $\theta_{13} \simeq 0$, as suggested by data. Depending on the values of the remaining non-vanishing parameters, we can distinguish two limits: $\left|P_{13}\right| \ll\left|P_{23}\right|$ and $\left|P_{23}\right| \ll$ $\left|P_{13}\right|$. The structure of the $P$-matrix is different in each case, and reads:

$$
\begin{aligned}
& P \simeq\left|P_{23}\right|\left(\begin{array}{ccc}
\lambda / \sqrt{6} & 0 & -\lambda \\
0 & 1 & 1 \\
-\lambda & 1 & 1
\end{array}\right), \text { for } \lambda=\frac{\left|P_{13}\right|}{\left|P_{23}\right|} \ll 1, \\
& P \simeq\left|P_{13}\right|\left(\begin{array}{ccc}
1 / \sqrt{6} & 0 & -1 \\
0 & \lambda & \lambda \\
-1 & \lambda & \sqrt{6}
\end{array}\right), \text { for } \lambda=\frac{\left|P_{23}\right|}{\left|P_{13}\right|} \ll 1 .
\end{aligned}
$$

In this approximation there are no phases in $P$, although there could be phases in the neutrino mass matrix. Let us analyze first the case where the neutrino mass matrix is also real, and later on, the general case allowing complex parameters.

\subsection{Real case}

The reconstruction of the high-energy parameters in terms of the low energy parameters is straightforward using eq.(9). The reconstructed high energy parameters in each limit read:

- $\left|P_{13}\right| \ll\left|P_{23}\right|$

$$
\begin{aligned}
M_{1} & \simeq 2 \sqrt{\frac{2}{3}} \frac{\left|P_{13}\right|}{m_{2}}\left\langle H_{u}^{0}\right\rangle^{2}, \\
M_{2} & \simeq \frac{2\left|P_{23}\right|}{m_{3}}\left\langle H_{u}^{0}\right\rangle^{2}, \\
\cos 2 z & \simeq 1 .
\end{aligned}
$$


so that the reconstructed Yukawa coupling is:

$$
\mathbf{Y}_{\nu} \simeq \sqrt{\left|P_{23}\right|}\left(\begin{array}{ccc}
\sqrt{\frac{\left|P_{13}\right|}{\sqrt{6}\left|P_{23}\right|}} & \sqrt{\sqrt{\frac{3}{8}} \frac{\left|P_{13}\right|}{\left|P_{23}\right|}} & -\sqrt{\sqrt{\frac{3}{8}} \frac{\left|P_{13}\right|}{\left|P_{23}\right|}} \\
-\frac{\left|P_{13}\right|}{2\left|P_{23}\right|} & 1 & 1
\end{array}\right)
$$

It is interesting that in this limit the lightest right-handed mass is essentially determined by the rate for the process $\tau \rightarrow e \gamma$, while the heaviest one, by the process $\tau \rightarrow \mu \gamma$. On the other hand, the complex angle in $R$ is such that in this case $\cos 2 z$ is very close to one, independently of the values of $\left|P_{13}\right|$ and $\left|P_{23}\right|$, as long as $\left|P_{13}\right| \ll\left|P_{23}\right|$.

- $\left|P_{23}\right| \ll\left|P_{13}\right|$

$$
\begin{aligned}
M_{1} & \simeq \frac{8\left|P_{23}\right|}{3 m_{2}+4 m_{3}}\left\langle H_{u}^{0}\right\rangle^{2}, \\
M_{2} & \simeq \frac{\left(3 m_{2}+4 m_{3}\right)\left|P_{13}\right|}{\sqrt{6} m_{2} m_{3}}\left\langle H_{u}^{0}\right\rangle^{2}, \\
\cos 2 z & \simeq \frac{3 m_{2}-4 m_{3}}{3 m_{2}+4 m_{3}},
\end{aligned}
$$

for the case in which the light neutrinos have the same $\mathrm{CP}$ parities, i.e. when $\phi=0$, and

$$
\begin{aligned}
M_{1} & \simeq \frac{8\left|P_{23}\right|}{-3 m_{2}+4 m_{3}}\left\langle H_{u}^{0}\right\rangle^{2}, \\
M_{2} & \simeq \frac{\left(-3 m_{2}+4 m_{3}\right)\left|P_{13}\right|}{\sqrt{6} m_{2} m_{3}}\left\langle H_{u}^{0}\right\rangle^{2}, \\
\cos 2 z & \simeq \frac{3 m_{2}+4 m_{3}}{3 m_{2}-4 m_{3}},
\end{aligned}
$$

when they have opposite CP parities, $\phi=\pi$.

The reconstructed Yukawa coupling is in this case:

$$
\mathbf{Y}_{\nu} \simeq \sqrt{\sqrt{6}\left|P_{13}\right|}\left(\begin{array}{ccc}
\sqrt{\frac{\sqrt{6}\left|P_{23}\right|}{\left|P_{13}\right|}} \frac{m_{2}}{3 m_{2}+4 m_{3}} & \sqrt{\frac{\left|P_{23}\right|}{\sqrt{6}\left|P_{13}\right|}} & \sqrt{\frac{\left|P_{23}\right|}{\sqrt{6}\left|P_{13}\right|}} \frac{-3 m_{2}+4 m_{3}}{3 m_{2}+4 m_{3}} \\
-\frac{1}{\sqrt{6}} & \frac{\sqrt{6} m_{2}}{3 m_{2}+4 m_{3}} \frac{\left|P_{23}\right|}{\left|P_{13}\right|} & 1
\end{array}\right)
$$

when $\phi=0$ and

$$
\mathbf{Y}_{\nu} \simeq \sqrt{\sqrt{6}\left|P_{13}\right|}\left(\begin{array}{ccc}
-\sqrt{\frac{\sqrt{6}\left|P_{23}\right|}{\left|P_{13}\right|}} \frac{m_{2}}{-3 m_{2}+4 m_{3}} & \sqrt{\frac{\left|P_{23}\right|}{\sqrt{6}\left|P_{13}\right|}} & \sqrt{\frac{\left|P_{23}\right|}{\sqrt{6}\left|P_{13}\right|}} \frac{3 m_{2}+4 m_{3}}{-3 m_{2}+4 m_{3}} \\
-\frac{i}{\sqrt{6}} & \frac{-i \sqrt{6} m_{2}}{-3 m_{2}+4 m_{3}} \frac{\left|P_{23}\right|}{\left|P_{13}\right|} & i
\end{array}\right)
$$

when $\phi=\pi$. 
In the case with $\left|P_{23}\right| \ll\left|P_{13}\right|$, the behaviour is opposite to the previous one: the lightest right-handed mass is determined by $\tau \rightarrow \mu \gamma$, and the heaviest by $\tau \rightarrow e \gamma$. In this limit, $\cos 2 z$ is also independent of $\left|P_{13}\right|$ and $\left|P_{23}\right|$, and takes a negative value, $\cos 2 z \simeq-0.75$, for $\phi=0$ and positive, $\cos 2 z \simeq-1.33$, for $\phi=\pi$.

The numerical results for the case with $\phi=0$ are shown in Fig 1 for different values of $\left|P_{13}\right|$ and $\left|P_{23}\right|$, where the two limits can be clearly distinguished. (Recall that for slepton masses of $\sim 400 \mathrm{GeV}$ and $\tan \beta=10,\left|P_{13,23}\right|<0.09$, however, we show the results for $\left|P_{13,23}\right|<1$ to allow for larger slepton masses or smaller values of $\tan \beta$.) In these figures, we have used the central values for the measured masses and mixing angles in [16], namely $m_{2}=8.9 \times 10^{-3} \mathrm{eV}, m_{3}=4.7 \times 10^{-2} \mathrm{eV}, \sin ^{2} \theta_{12}=0.30$ and $\sin ^{2} \theta_{23}=0.50$. These parameters have to be run from the electroweak scale to the decoupling scale [51] and this introduces corrections smaller than a $60 \%$ on the reconstructed parameters. In addition, the experimental error on the low energy parameters introduces an indeterminacy on the reconstructed parameters smaller than a factor of two, that will be reduced in forthcoming experiments. It is apparent from this analysis that the observation of rare decays would be an important step towards reconstructing the complete Lagrangian of the $2 \mathrm{RHN}$ model.

\subsection{Complex case, $\theta_{13}=0$}

For the complex case, we will show first the results for $\theta_{13}=0$, so that the high-energy parameters will depend only on the Majorana phase $\phi$ (recall that in this case $P$ is real with a good approximation). Later on, we will discuss the situation with nonvanishing $\theta_{13}$. On the other hand, for the atmospheric and solar angles, we maintain the experimentally favoured values $\theta_{23} \simeq \pi / 4, \theta_{12} \simeq \pi / 6$. The analytical expressions for the high energy parameters are given in this case by:

$$
\text { - }\left|P_{13}\right| \ll\left|P_{23}\right|
$$

$$
\begin{aligned}
M_{1} & \simeq 2 \sqrt{\frac{2}{3}} \frac{\left|P_{13}\right|}{m_{2}}\left\langle H_{u}^{0}\right\rangle^{2}, \\
M_{2} & \simeq \frac{2\left|P_{23}\right|}{m_{3}}\left\langle H_{u}^{0}\right\rangle^{2}, \\
\cos 2 z & \simeq 1 .
\end{aligned}
$$



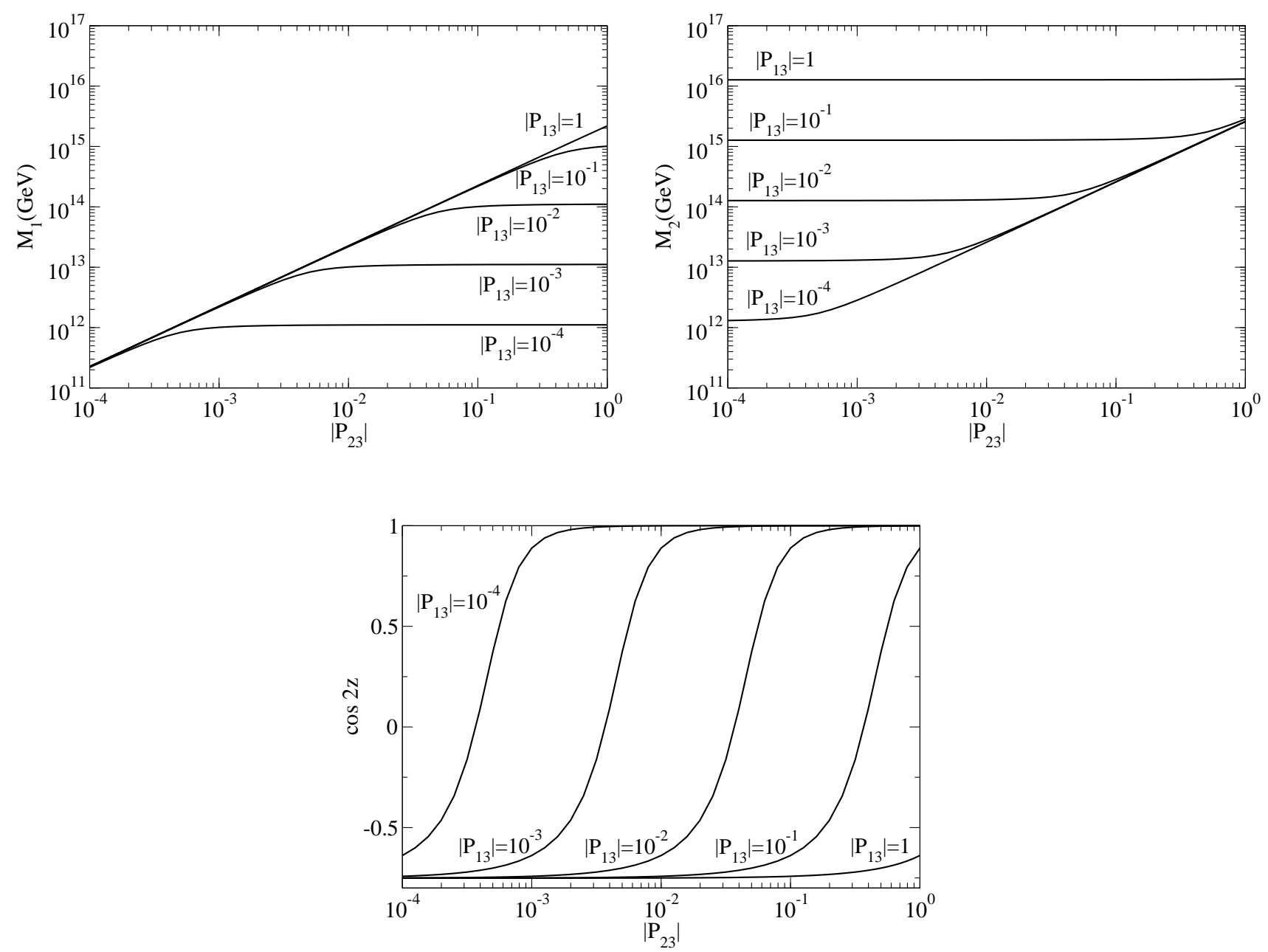

Figure 1: Reconstructed right-handed neutrino masses and parameter $z$ in the matrix $R$, for different values of $\left|P_{13}\right|$ and $\left|P_{23}\right|$, in the limit $\left|P_{12}\right| \ll\left|P_{13}\right|,\left|P_{23}\right|$. Here, we have taken $\theta_{23} \simeq \pi / 4, \theta_{12} \simeq \pi / 6, \theta_{13} \simeq 0$ and $\phi \simeq 0$ (so that all the parameters involved in the reconstruction procedure are real to a good approximation).

Therefore, the Yukawa coupling is:

$$
\mathbf{Y}_{\nu} \simeq \sqrt{\left|P_{23}\right|}\left(\begin{array}{ccc}
\sqrt{\frac{\left|P_{13}\right|}{\sqrt{6}\left|P_{23}\right|}} e^{i \phi / 2} & \sqrt{\sqrt{\frac{3}{8}} \frac{\left|P_{13}\right|}{\left|P_{23}\right|} e^{i \phi / 2}} & -\sqrt{\sqrt{\frac{3}{8}} \frac{\left|P_{13}\right|}{\left|P_{23}\right|}} e^{i \phi / 2} \\
-\frac{\left|P_{13}\right|}{2\left|P_{23}\right|} & 1 & 1
\end{array}\right)
$$

Note that although in this limit the right-handed masses and $\cos 2 z$ do not depend on the Majorana phase, the Yukawa coupling does. The dependence on the phase results from the phase in the leptonic mixing matrix, $U$, that enters in the parametrization of the Yukawa coupling, eq.(1). Similarly to the real case, the lightest right-handed mass is essentially determined by the rate for the process $\tau \rightarrow e \gamma$, while the heaviest one, by the process $\tau \rightarrow \mu \gamma$. 
- $\left|P_{23}\right| \ll\left|P_{13}\right|$

$$
\begin{aligned}
M_{1} & \simeq \frac{8\left|P_{23}\right|\left\langle H_{u}^{0}\right\rangle^{2}}{\sqrt{9 m_{2}^{2}+16 m_{3}^{2}+24 m_{2} m_{3} \cos \phi}}, \\
M_{2} & \simeq \frac{\left|P_{13}\right| \sqrt{9 m_{2}^{2}+16 m_{3}^{2}+24 m_{2} m_{3} \cos \phi}}{\sqrt{6} m_{2} m_{3}}\left\langle H_{u}^{0}\right\rangle^{2}, \\
\cos 2 z & \simeq \frac{3 m_{2} e^{i \phi}-4 m_{3}}{3 m_{2} e^{i \phi}+4 m_{3}},
\end{aligned}
$$

so that the reconstructed Yukawa coupling is:

$$
\mathbf{Y}_{\nu} \simeq \sqrt{\sqrt{6} \frac{\Delta}{\Delta^{*}}\left|P_{13}\right|}\left(\begin{array}{ccc}
\sqrt{\frac{\sqrt{6}\left|P_{23}\right|}{\left|P_{13}\right|} \frac{m_{2} e^{i \phi}}{|\Delta|^{2}}} & \sqrt{\frac{\left|P_{23}\right|}{\sqrt{6}\left|P_{13}\right|}} \frac{\Delta^{*}}{\Delta} & \sqrt{\frac{\left|P_{23}\right|}{\sqrt{6}\left|P_{13}\right|}} \frac{-3 m_{2} e^{i \phi}+4 m_{3}}{|\Delta|^{2}} \\
-\frac{e^{i \phi / 2}}{\sqrt{6}} & \frac{\sqrt{6} m_{2} e^{-i \phi / 2}}{\Delta^{2}} \frac{\left|P_{23}\right|}{\left|P_{13}\right|} & e^{i \phi / 2}
\end{array}\right)
$$

where $\Delta=\sqrt{3 m_{2} e^{-i \phi}+4 m_{3}}$. Contrary to the previous limit, here the righthanded masses and $R$ do depend on the Majorana phase. Concerning which processes are relevant to determine which parameter, the conclusion is analogous to the real case: the lightest right-handed mass is essentially determined by the process $\tau \rightarrow \mu \gamma$, and the heaviest by $\tau \rightarrow e \gamma$.

The numerical results can be found in Fig [2 We see that when $\left|P_{13}\right| \ll\left|P_{23}\right|$ the correlation between the right-handed masses and $\left|P_{13}\right|$ or $\left|P_{23}\right|$ is very tight, allowing a very precise reconstruction of the high-energy parameters. However, when $\left|P_{23}\right| \ll\left|P_{13}\right|$ the reconstruction is more complicated, and the precise determination of the highenergy parameters would require the measurement of the Majorana phase. In Fig 2 we have sampled $\phi$ between 0 and $2 \pi$ and show the regions at $2 \sigma$ from the main value. These regions are fairly narrow, so even without knowing the Majorana phase, it could be possible to reconstruct the right-handed masses from rare decays, up to a factor of two.

On the other hand, for the numerical value of $\cos 2 z$ we show both the absolute value and the argument as a function of the Majorana phase, $\phi$, for fixed $\left|P_{23}\right|=10^{-2}$ and for different values of $\left|P_{13}\right|$. It can be checked that the prediction for $\cos 2 z$ depends on the ratio $\left|P_{13}\right| /\left|P_{23}\right|$, so the results for other values of $\left|P_{23}\right|$ could be read easily from this figure. We find that when $\left|P_{13}\right| \ll\left|P_{23}\right|$ both the absolute value and the argument of $\cos 2 z$ are not very sensitive to $\phi$. However, despite the matrix $R$ depends weakly on $\phi$, it is not possible to reconstruct the Yukawa coupling, due to the dependence of the Yukawa matrix with $\phi$ through the leptonic mixing matrix in eq.(11). On the 

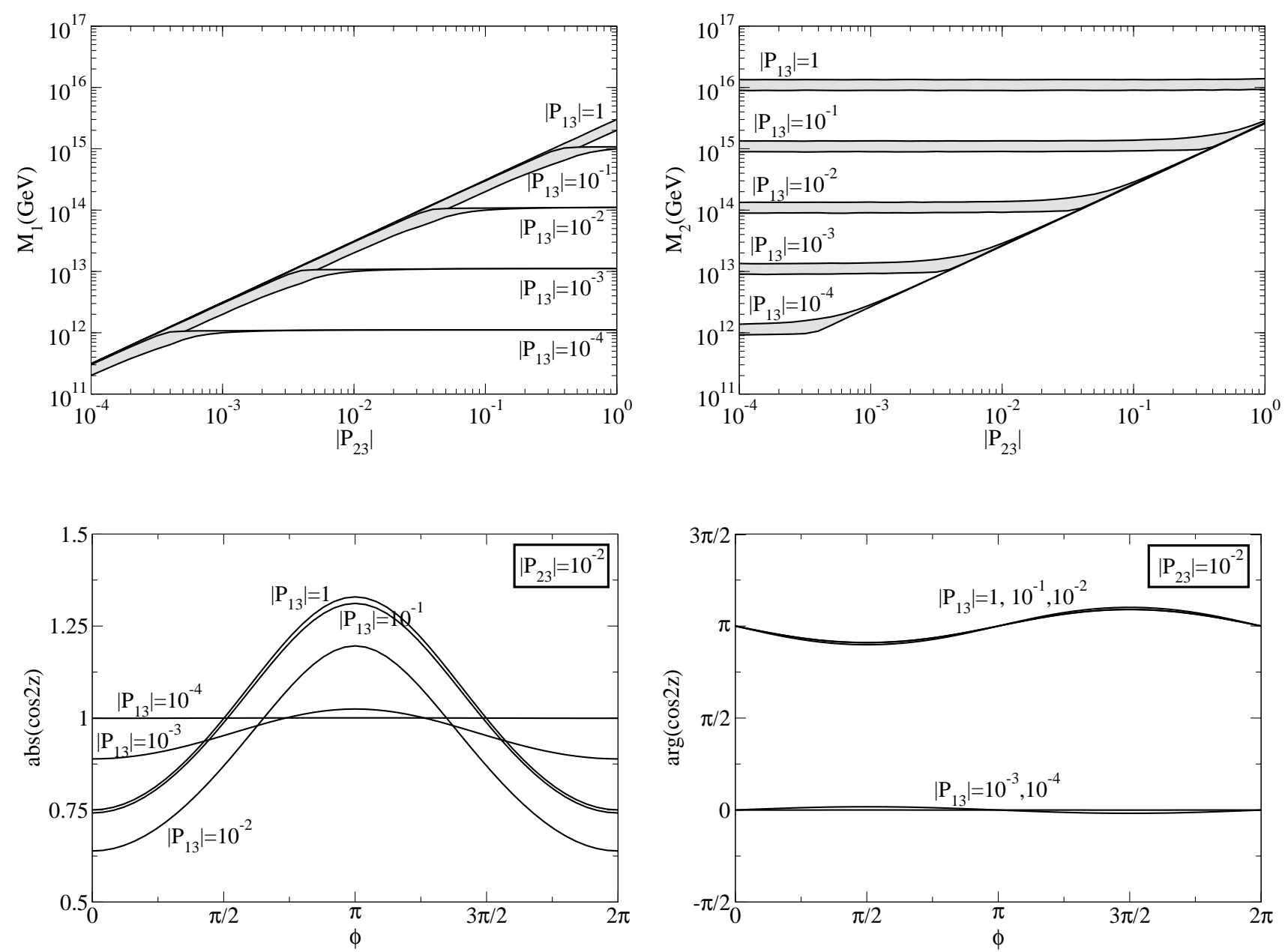

Figure 2: The same as Fig 1, but sampling over different values for $\phi$. The shaded areas represent the regions at $2 \sigma$ from the main value. For $\cos 2 z$ we plot both the absolute value and the argument as a function of the Majorana phase $\phi$, for fixed $\left|P_{23}\right|=10^{-2}$ and different values of $\left|P_{13}\right|$.

other hand, when $\left|P_{23}\right| \ll\left|P_{13}\right|$, the absolute value of $\cos 2 z$ depends strongly on $\phi$. Therefore, in both cases the reconstruction of the Yukawa coupling would require the determination of the Majorana phase, although it would not be necessary for a rough reconstruction of the right-handed masses.

\subsection{Complex case, $\theta_{13} \neq 0$}

Finally we show the results including the effects of $\theta_{13}$ and the phase $\delta$. Although it is possible to derive analytic expressions for the high-energy parameters in the different limits, the expressions are very complicated and difficult to analyze. Therefore, in this subsection we will limit ourselves to show the numerical results, that can be found in 
Fig 3

We find that in the whole parameter space, the measurement of $\theta_{13}, \delta$ and $\phi$ would be desirable for a precise determination of the right-handed masses. However, even without knowing these parameters, the determination of the rates for the rare lepton decays would allow the reconstruction of the right-handed masses up to a factor of three.

On the other hand, when $\theta_{13}$ is large, the reconstruction of the parameter $z$ necessarily requires the measurement of all the low-energy parameters. The situation is particularly critical when $\left|P_{13}\right| \ll\left|P_{23}\right|$, since $\cos 2 z$ is very sensitive to the Dirac phase (when $\theta_{13}$ is large). For instance, when $\theta_{13}=0.1$, the absolute value of $\cos 2 z$ can vary between 0.6 and 1.5 when $\delta$ and $\phi$ vary between 0 and $2 \pi$. When $\left|P_{23}\right| \ll\left|P_{13}\right|$, the dispersion produced by the angle $\theta_{13}$ is smaller, between 0.8 and 1.3 . Concerning the argument of $\cos 2 z$ the range of values is smaller, although still important, particularly in the limit $\left|P_{13}\right| \ll\left|P_{23}\right|$. Therefore, when $\theta_{13}$ is large, it seems unavoidable the precise measurement of $\theta_{13}, \delta$ and $\phi$ to determine $\cos 2 z$ and to reconstruct the Yukawa coupling.

\section{The $2 \mathrm{RHN}$ model as a limit of the $3 \mathrm{RHN}$ model}

Although the 2RHN model can explain all the neutrino experiments, the 3RHN model is without any doubt much more appealing from the theoretical point of view. Nevertheless, there are some situations where the $3 \mathrm{RHN}$ model can be well approximated by a 2 RHN model, both from the point of view of neutrino masses, as from the point of view of radiative corrections, parametrized by the matrix $P$. In these situations, the procedure presented in this paper to reconstruct the high-energy parameters would apply.

Let us discuss first the case of the neutrino mass matrix, that in the basis where the right-handed neutrino mass matrix is diagonal reads:

$$
\mathcal{M}_{i j}=\frac{y_{1 i} y_{1 j}}{M_{1}}+\frac{y_{2 i} y_{2 j}}{M_{2}}+\frac{y_{3 i} y_{3 j}}{M_{3}}
$$

where $y_{i j}=\left(\mathbf{Y}_{\nu}\right)_{i j}$. Two right-handed neutrinos dominate the see-saw when

$$
\frac{y_{1 i} y_{1 j}}{M_{1}} \ll \frac{y_{2 i} y_{2 j}}{M_{2}}, \frac{y_{3 i} y_{3 j}}{M_{3}} \text { or }
$$



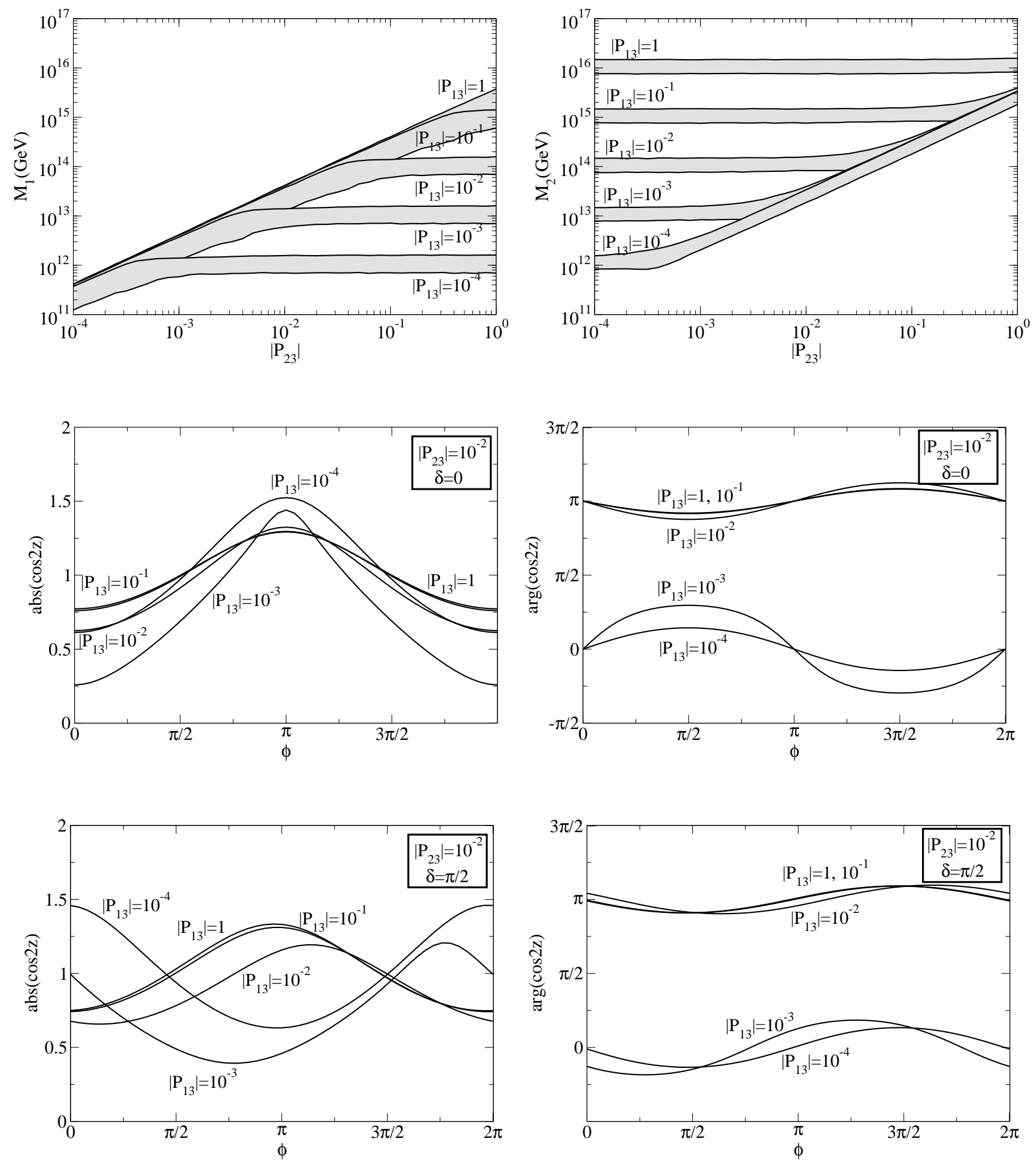

Figure 3: The same as Fig 1 but sampling over different values for $\phi$ and $\delta$ for $\theta_{13}=0.1$. The shaded areas represent the regions at $2 \sigma$ from the main value. For $\cos 2 z$ we plot both the absolute value and the argument as a function of the Majorana phase $\phi$, for fixed $\left|P_{23}\right|=10^{-2}$ and different values of $\left|P_{13}\right|$, and for two values of the phase $\delta: 0$ and $\pi / 2$. 


$$
\begin{aligned}
& \frac{y_{2 i} y_{2 j}}{M_{2}} \ll \frac{y_{1 i} y_{1 j}}{M_{1}}, \frac{y_{3 i} y_{3 j}}{M_{3}} \quad \text { or } \\
& \frac{y_{3 i} y_{3 j}}{M_{3}} \ll \frac{y_{1 i} y_{1 j}}{M_{1}}, \frac{y_{2 i} y_{2 j}}{M_{2}} \quad \text { for all } i, j=1,2,3 .
\end{aligned}
$$

The most interesting cases are the first and the third. The first one corresponds to the case in which the Yukawa couplings for the first generation of right handed neutrinos are tiny, $y_{1 i} \ll y_{2 i}, y_{3 i}$, for $i=1,2$, 3 . If this is the case, the radiative corrections are also dominated by the same two right-handed neutrinos, the two heaviest ones. Therefore, in this case the $3 \mathrm{RHN}$ model can be well approximated by a $2 \mathrm{RHN}$ model, both from the point of view of neutrino masses as of radiative corrections. Since the two relevant right-handed neutrinos are the two heaviest ones, the corresponding Yukawa couplings could be large, and the radiative corrections could be sizable. ${ }^{4}$

The third case corresponds to the situation where the mass of the heaviest righthanded neutrino is much larger than the mass of the other two, $M_{3} \gg M_{1}, M_{2}[12$. However, in general the heaviest right-handed neutrino will produce sizable contributions to the radiative corrections. If this is the case, the $3 \mathrm{RHN}$ model could be reduced to a $2 \mathrm{RHN}$ model only from the point of view of neutrino masses, but not from the point of view of the radiative corrections. Nevertheless, there are some circumstances in which the heaviest right-handed neutrino indeed does not contribute to the radiative corrections and does not leave any imprint in $P$, so that this matrix is only determined by the Yukawa couplings of the two lightest generations of right-handed neutrinos. If this occurs, the $3 \mathrm{RHN}$ model would also be well approximated by a 2RHN model from the point of view of the radiative corrections. This situation arises for example when the mass of the heaviest right-handed neutrino is very close to the Planck mass, although this possibility seems a bit contrived.

A more plausible situation arises in models with gauge mediated supersymmetry breaking [52. So far, we have implicitly assumed that the boundary conditions for the soft breaking terms are set at the Planck scale. However, if the mass of the messenger particles involved in the supersymmetry breaking mechanism is smaller than $M_{3}$ but larger than $M_{2}$, then the heaviest right-handed neutrino would decouple at an energy larger than the energy at which supersymmetry breaking is communicated to the

\footnotetext{
${ }^{4}$ In this scenario there would be no relation whatsoever between leptogenesis and low energy observables: leptogenesis would depend on the couplings of the lightest right-handed neutrino, whereas neutrino and slepton parameters would be determined by the couplings of the two heaviest righthanded neutrinos.
} 
observable sector. Consequently, it would not participate in the radiative corrections of the parameters of the Lagrangian. If this is the case, only the two lightest righthanded neutrinos would contribute to the radiative corrections and to the neutrino mass generation, and therefore the $3 \mathrm{RHN}$ model could be well approximated by a 2RHN model.

The experimental signature of this scenario would be a light gravitino, although probably not ultra-light, since the mass of the messenger has to be larger than the mass of the next-to-lightest right-handed neutrino, that is expected to be rather large. The gravitino mass in these scenarios can be estimated as 53

$$
m_{3 / 2}=\frac{F}{\sqrt{3} M_{P}} \sim \frac{\pi}{\sqrt{3} \alpha} \frac{M_{\mathrm{mes}}}{M_{P}} \widetilde{m},
$$

where we have assumed vanishing cosmological constant and $M_{P}=\left(8 \pi G_{N}\right)^{-1 / 2}=$ $2.4 \times 10^{18} \mathrm{GeV}$ is the reduced Planck mass. In this formula, $F$ measures supersymmetry breaking in the messenger sector, $M_{\text {mes }}$ is the mass of the messenger particles and $\widetilde{m} \sim(\alpha / \pi) F / M_{\text {mes }}$ is the typical soft mass scale (recall that in gauge mediated supersymmetry breaking scenarios gaugino masses are generated at one loop and scalar masses at two loops). If only the heaviest right-handed neutrino decouples from the radiative corrections, it has to happen that $M_{2}<M_{\text {mes }}<M_{3}$. So, if the gravitino mass is measured, a lower bound on $M_{3}$ would follow:

$$
M_{3} \gtrsim \frac{\sqrt{3} \alpha}{\pi} \frac{m_{3 / 2}}{\widetilde{m}} M_{P}
$$

Alternatively, if $M_{2}$ can be reconstructed from low energy data, a lower bound on the gravitino mass would follow. Note that the precise determination of $M_{2}$ requires the knowledge of the cut-off scale, that is not known a priori. However one could compute $M_{2}$ assuming that the cut-off is set at the Planck scale instead of the actual messenger scale. Then, the value obtained for $M_{2}$ would be smaller (see the Appendix for details) and therefore the bound on the gravitino mass obtained in this way would hold, although it would be more conservative than the actual bound.

Besides, when supersymmetry breaking is mediated to the observable sector through gauge interactions by particles with a mass smaller than the Planck mass, the soft terms are almost proportional to the identity at the cut-off scale. Thus, any flavour changing effect or mass splitting between sleptons or sneutrinos observed at low energies would be entirely due to radiative corrections. On the other hand, $\mathrm{CP}$ violating effects could 
originate in other sectors, such as the neutralino or the chargino sector, although there could be a contribution from $P$ that might be disentangled.

Finally, if in addition the gravitino is the lightest supersymmetric particle, some interesting cosmological consequences would follow. For instance, the strong bounds on the reheating temperature, $T_{R} \lesssim 10^{6} \mathrm{GeV}$ for $m_{3 / 2} \sim \mathcal{O}(1 \mathrm{TeV}$ ) [54], would be relaxed so that it could be as large as $10^{11} \mathrm{GeV}$ [55]. This has crucial consequences for the leptogenesis mechanism, since the mass of the lightest right-handed could be compatible with the constraints on the reheating temperature from preventing gravitino overproduction 4756 .

\section{Conclusions}

The two right-handed neutrino model can explain all the neutrino oscillation experiments, but depends on less parameters than the conventional three right-handed neutrino model. Therefore, the serious problem of the lack of predictivity of the conventional see-saw model is softened. In this paper we have exploited this observation to argue that the high-energy parameters of the two right-handed neutrino model could be reconstructed using just low energy experiments, provided supersymmetry is discovered and some hypotheses are made about the structure of the soft terms at the cut-off scale.

To this end, we have proposed an alternative parametrization of the two righthanded neutrino model just in terms of low energy observables, namely the neutrino mass matrix and the off-diagonal elements of the matrix $\mathbf{Y}_{\nu}^{\dagger} \mathbf{Y}_{\nu}$, that is responsible for the radiative corrections of the slepton parameters, and that in particular induces rare leptonic decays. We have discussed the present information available on these parameters and the prospects to improve our knowledge of them in the next few years. Except for the case of the Majorana phase, we find the prospects very encouraging.

We have presented an exact procedure that allows to reconstruct the high-energy superpotential of the two right-handed neutrino model in terms of the low energy parameters. We have applied this procedure to a particular scenario with $B R(\mu \rightarrow$ $e \gamma) \ll B R(\tau \rightarrow(\mu, e) \gamma)$, and we have found that in the case where all the parameters are real and $\theta_{13}$ is small, the detection of the rare decays $\tau \rightarrow \mu \gamma$ and $\tau \rightarrow e \gamma$ would be a very important step towards reconstructing the high-energy parameters. We have 
also analyzed the impact of the phases in the leptonic mixing matrix on this conclusion, since they could be the worst determined among all the low energy parameters. We have found that when the angle $\theta_{13}$ is large and the phases in the leptonic mixing matrix do not vanish, the connection is more diffuse, although it could still be possible to determine the right-handed masses up to a factor of three. The reconstruction of the neutrino Yukawa coupling is more complicated, since in general would require the measurement of $\theta_{13}, \delta$ and $\phi$.

Finally, we have argued that this procedure does not apply only to the strict two right-handed neutrino model. There are limits of the three right-handed neutrino model that resemble a two right-handed neutrino model to a good approximation, both from the point of view of neutrino masses, as from the point of view of radiative corrections. In these limits, the procedure proposed in this paper applies.

\section{Acknowledgments}

I would like to thank Alberto Casas, and especially Sacha Davidson for very interesting discussions. I would also like to thank the CERN Theory Division for hospitality during the last stages of this work.

\section{A Appendix}

Throughout the paper we have used as parameters to reconstruct the high-energy theory $P=\mathbf{Y}_{\nu}^{\dagger} \mathbf{Y}_{\nu}$ and $\mathcal{M}=\mathbf{Y}_{\nu}^{T} \operatorname{diag}\left(M_{1}^{-1}, M_{2}^{-1}\right) \mathbf{Y}_{\nu}\left\langle H_{u}^{0}\right\rangle^{2}$. However, to disentangle $P$ it is necessary to know not only the cut-off scale but also the decoupling scales, that are not known a priori. Nevertheless, the procedure can be applied recursively in order to reconstruct the high-energy parameters.

In this Appendix it will be shown that this recursive procedure could be avoided using as low energy parameters of the $2 \mathrm{RHN}$ model

$$
\begin{aligned}
\mathcal{M} & =\mathbf{Y}_{\nu}^{T} \operatorname{diag}\left(M_{1}^{-1}, M_{2}^{-1}\right) \mathbf{Y}_{\nu}\left\langle H_{u}^{0}\right\rangle^{2} \\
P & =\mathbf{Y}_{\nu}^{\dagger} \operatorname{diag}\left(\log \frac{\Lambda}{M_{1}}, \log \frac{\Lambda}{M_{2}}\right) \mathbf{Y}_{\nu},
\end{aligned}
$$

where the second parameter is the combination that appears in the leading-log approx-

imation to the solution of the renormalization group equations, and $\Lambda$ is the cut-off scale. 
Defining the new parameters

$$
\begin{aligned}
\widetilde{\mathbf{Y}}_{\nu} & =\operatorname{diag}\left(\sqrt{\log \frac{\Lambda}{M_{1}}}, \sqrt{\log \frac{\Lambda}{M_{2}}}\right) \mathbf{Y}_{\nu}, \\
\widetilde{M}_{1} & =M_{1} \log \frac{\Lambda}{M_{1}} \\
\widetilde{M}_{2} & =M_{2} \log \frac{\Lambda}{M_{1}}
\end{aligned}
$$

and substituting in eq.(30), one finds that

$$
\begin{aligned}
\mathcal{M} & =\widetilde{\mathbf{Y}}_{\nu}^{T} \operatorname{diag}\left(\widetilde{M}_{1}^{-1}, \widetilde{M}_{2}^{-1}\right) \widetilde{\mathbf{Y}}_{\nu}\left\langle H_{u}^{0}\right\rangle^{2}, \\
P & =\widetilde{\mathbf{Y}}_{\nu}^{\dagger} \widetilde{\mathbf{Y}}_{\nu} .
\end{aligned}
$$

Therefore, we can apply the general formulas eq.(17) to solve for the parameters $\widetilde{\mathbf{Y}}_{\nu}$, $\widetilde{M}_{1}$ and $\widetilde{M}_{2}$. Finally, inverting eq.(31) we can reconstruct the actual high-energy parameters $\mathbf{Y}_{\nu}, M_{1}$ and $M_{2}$. Note that there are always two solutions for $M_{1}$ and $M_{2}$, one larger than $\Lambda / e$ and the other smaller.

From eq. (31) it is possible to estimate the impact of our ignorance of the cut-off scale on the reconstructed values of the right-handed masses. From the reconstruction procedure it is always possible to compute $\widetilde{M}_{1}$ and $\widetilde{M}_{2}$, and from eq.(31), the actual masses $M_{1}$ and $M_{2}$, that would depend on the cut-off scale. If we set the cut-off scale at the Planck mass, we would obtain $M_{1}\left(M_{P}\right)$ and $M_{2}\left(M_{P}\right)$. However, it could happen that the actual cut-off scale is smaller than the Planck mass, for instance in models with gauge mediated supersymmetry breaking; in that case we would obtain $M_{1}(\Lambda)$ and $M_{2}(\Lambda)$. In Fig 4 we compare the values of the masses computed assuming that the cut-off is set at the Planck mass or at a different cut-off, for fixed $\widetilde{M}_{1}$ or $\widetilde{M}_{2}$. We find that taking as the cut-off the Planck mass instead of an intermediate scale underestimates the value of the reconstructed right-handed masses. Nevertheless, the error made is usually smaller than one order of magnitude.

\section{References}

[1] P. Minkowski, Phys. Lett. B 67 (1977) 421. M. Gell-Mann, P. Ramond and R. Slansky, Proceedings of the Supergravity Stony Brook Workshop, New York 1979, eds. P. Van Nieuwenhuizen and D. Freedman; T. Yanagida, Proceedinds of the Workshop on Unified Theories and Baryon Number in the Universe, Tsukuba, Japan 1979, 


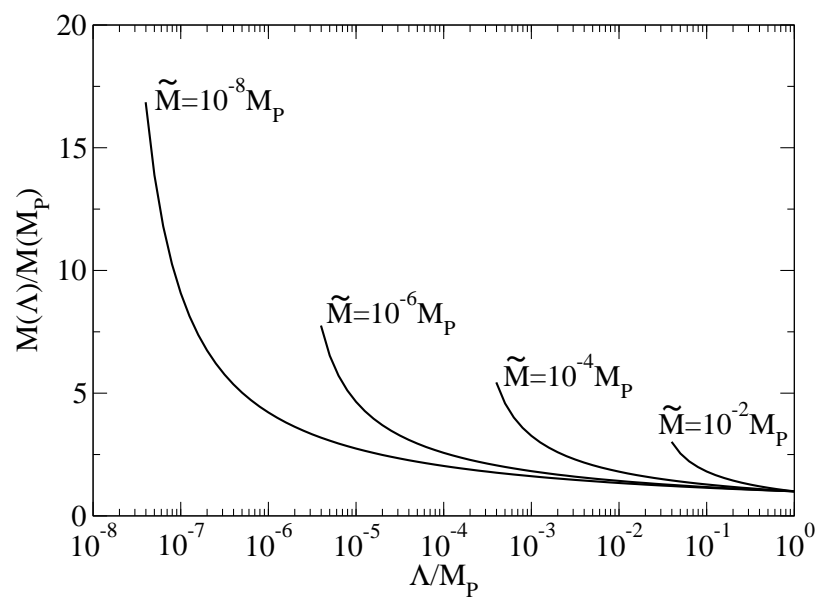

Figure 4: Relation between the right-handed neutrino mass reconstructed using the actual cut-off scale $\Lambda$, and reconstructed using the Planck mass as cut-off, for different values of $\widetilde{M}$ (see details in the Appendix).

eds. A. Sawada and A. Sugamoto; R. N. Mohapatra, G. Senjanovic, Phys.Rev.Lett. 44 (1980)912, ibid. Phys.Rev. D23 (1981) 165; S. L. Glashow, The Future Of Elementary Particle Physics, In ${ }^{*}$ Cargese 1979, Proceedings, Quarks and Leptons*, 687-713 and Harvard Univ.Cambridge - HUTP-79-A059 (79,REC.DEC.) 40p. J. Schechter and J. W. F. Valle, Phys. Rev. D 22 (1980) 2227.

[2] A. Broncano, M. B. Gavela and E. Jenkins, Phys. Lett. B 552 (2003) 177

[3] F. Borzumati and A. Masiero, Phys. Rev. Lett. 57 (1986) 961;

[4] J. Hisano, T. Moroi, K. Tobe, M. Yamaguchi and T. Yanagida, Phys. Lett. B 357 (1995) 579; J. Hisano, T. Moroi, K. Tobe and M. Yamaguchi, Phys. Rev. D 53 (1996) 2442.

[5] J. A. Casas and A. Ibarra, Nucl. Phys. B 618 (2001) 171.

[6] S. Lavignac, I. Masina and C. A. Savoy, Phys. Lett. B 520 (2001) 269. S. Lavignac, I. Masina and C. A. Savoy, Nucl. Phys. B 633 (2002) 139 arXiv:hep-ph/0202086.

[7] A. Romanino and A. Strumia, Nucl. Phys. B 622 (2002) 73 J. R. Ellis, J. Hisano, S. Lola and M. Raidal, Nucl. Phys. B 621 (2002) 208 
[8] H. Baer, C. Balazs, J. K. Mizukoshi and X. Tata, Phys. Rev. D 63 (2001) 055011 G. A. Blair, W. Porod and P. M. Zerwas, Eur. Phys. J. C 27 (2003) 263 A. Freitas, W. Porod and P. M. Zerwas, arXiv:hep-ph/0509056.

[9] S. Davidson and A. Ibarra, JHEP 0109 (2001) 013

[10] J. R. Ellis, J. Hisano, M. Raidal and Y. Shimizu, Phys. Rev. D 66 (2002) 115013

[11] A. Kleppe, "Extending The Standard Model With Two Right-Handed Neutrinos", in *Lohusalu 1995, Neutrino physics*, 118-125; A. Kleppe, "Extending The Standard Model By Including Right-Handed Neutrinos". Prepared for Workshop on What Comes Beyond the Standard Model, Bled, Slovenia, 29 Jun - 9 Jul 1998;. E. Ma, D. P. Roy and U. Sarkar, Phys. Lett. B 444 (1998) 391; P. H. Frampton, S. L. Glashow and T. Yanagida, Phys. Lett. B 548 (2002) 119. M. Raidal and A. Strumia, Phys. Lett. B 553, 72 (2003). V. Barger, D. A. Dicus, H. J. He and T. j. Li, Phys. Lett. B 583 (2004) 173 W. l. Guo and Z. z. Xing, Phys. Lett. B 583 (2004) 163 R. Gonzalez Felipe, F. R. Joaquim and B. M. Nobre, Phys. Rev. D 70, 085009 (2004) S. Raby, Phys. Lett. B 561 (2003) 119; B. Dutta and R. N. Mohapatra, Phys. Rev. D 68 (2003) 056006; R. Kuchimanchi and R. N. Mohapatra, Phys. Lett. B 552 (2003) 198; T. Appelquist and R. Shrock, Phys. Lett. B 548 (2002) 204, Phys. Rev. Lett. 90 (2003) 201801; T. Endoh, S. Kaneko, S. K. Kang, T. Morozumi and M. Tanimoto, Phys. Rev. Lett. 89 (2002) 231601. S. Chang, S. K. Kang and K. Siyeon, Phys. Lett. B 597 (2004) 78

[12] A. Y. Smirnov, Phys. Rev. D 48 (1993) 3264; M. Jezabek and Y. Sumino, Phys. Lett. B 440 (1998) 327; S. F. King, Nucl. Phys. B 562 (1999) 57, Nucl. Phys. B 576 (2000) 85, JHEP 0209 (2002) 011; S. F. King and G. G. Ross, Phys. Lett. B 574 (2003) 239. W. Rodejohann, Eur. Phys. J. C 32 (2004) 235; H. K. Dreiner, H. Murayama and M. Thormeier, Nucl. Phys. B 729 (2005) 278

[13] Z. Maki, M. Nakagawa and S. Sakata, Prog. Theor. Phys. 28 (1962) 870.

[14] A. Ibarra and G. G. Ross, Phys. Lett. B 575 (2003) 279

[15] A. Ibarra and G. G. Ross, Phys. Lett. B 591 (2004) 285 
[16] M. Maltoni, T. Schwetz, M. A. Tortola and J. W. F. Valle, New J. Phys. 6 (2004) 122

[17] P. Aprili et al. [ICARUS Collaboration], "The ICARUS experiment: A secondgeneration proton decay experiment and CERN-SPSC-2002-027

[18] M. Guler et al. [OPERA Collaboration], CERN-SPSC-2000-028

[19] E. Ables et al. [MINOS Collaboration], FERMILAB-PROPOSAL-0875

[20] D. S. Ayres et al. [NOvA Collaboration], arXiv:hep-ex/0503053.

[21] Y. Itow et al., "The JHF-Kamioka neutrino project, arXiv:hep-ex/0106019.

[22] F. Ardellier et al., arXiv:hep-ex/0405032.

[23] S. Choubey and S. T. Petcov, Phys. Lett. B 594 (2004) 333

[24] A. Bandyopadhyay, S. Choubey, S. Goswami and S. T. Petcov, Phys. Rev. D 72 (2005) 033013

[25] P. Hernandez. "Towards precision lepton flavour physics". Talk given at What's $\nu$, IFT MiniWorkshop on Neutrino Physics 2005 , Madrid, Spain, May 2005.

[26] S. Pascoli, S. T. Petcov and T. Schwetz, arXiv:hep-ph/0505226.

[27] M. L. Brooks et al. [MEGA Collaboration], Phys. Rev. Lett. 83 (1999) 1521

[28] K. Abe et al. [Belle Collaboration], Phys. Rev. Lett. 92 (2004) 171802

[29] B. Aubert et al. [BABAR Collaboration], Phys. Rev. Lett. 95 (2005) 041802

[30] K. Hayasaka et al., Phys. Lett. B 613 (2005) 20

[31] A. Masiero, S. K. Vempati and O. Vives, New J. Phys. 6 (2004) 202

[32] A. Baldini, AIP Conf. Proc. 721 (2004) 289.

[33] P. Wintz In: H.V. Klapdor-Kleingrothaus and I.V. Krivosheina, Editors, Proceedings of the First International Symposium on Lepton and Baryon Number Violation, Institute of Physics, Bristol/Philadelphia (1998), p. 534. 
[34] A. van der Schaaf, Nucl. Instrum. Meth. A 503 (2003) 281.

[35] A. Sato, "PRIME PRISM Muon to Electron conversion experiment", talk given at NuFact05, Frascati, Italy, June 2005.

[36] J. Aysto et al., arXiv:hep-ph/0109217.

[37] A. G. Akeroyd et al. [SuperKEKB Physics Working Group Collaboration], arXiv:hep-ex/0406071. T. Iijima, "Overview of Physics at Super B-Factory", talk given at the 6th Workshop on a Higher Luminosity B Factory, KEK, Tsukuba, Japan, November 2004.

[38] B. C. Regan, E. D. Commins, C. J. Schmidt and D. DeMille, Phys. Rev. Lett. 88 (2002) 071805.

[39] D. Kawall, F. Bay, S. Bickman, Y. Jiang and D. DeMille, Phys. Rev. Lett. 92 (2004) 133007

[40] D. Kawall, F. Bay, S. Bickman, Y. Jiang and D. DeMille, AIP Conf. Proc. 698 (2004) 192.

[41] S. K. Lamoreaux, arXiv:nucl-ex/0109014.

[42] C. Y. Liu and S. K. Lamoreaux, Mod. Phys. Lett. A 19 (2004) 1235.

[43] D. A. Demir and Y. Farzan, arXiv:hep-ph/0508236.

[44] P. G. Harris et al., Phys. Rev. Lett. 82 (1999) 904.

[45] Y. K. Semertzidis et al., arXiv:hep-ph/0012087.

[46] K. Inami et al. [Belle Collaboration], Phys. Lett. B 551 (2003) 16

[47] S. Davidson and A. Ibarra, Phys. Lett. B 535, 25 (2002)

[48] P. H. Chankowski and K. Turzynski, Phys. Lett. B 570 (2003) 198

[49] M. Fukugita and T. Yanagida, Phys. Lett. B 174 (1986) 45. 
[50] Previous attempts to reconstruct the high energy lagrangian from low energy observables can be found in refs. 2910, as well as in S. Davidson, JHEP 0303, 037 (2003); E. K. Akhmedov, M. Frigerio and A. Y. Smirnov, JHEP 0309 (2003) 021 ;

[51] K. S. Babu, C. N. Leung and J. T. Pantaleone, Phys. Lett. B 319, 191 (1993); P. H. Chankowski and Z. Pluciennik, Phys. Lett. B 316 (1993) 312; J. R. Ellis and S. Lola, Phys. Lett. B 458 (1999) 310; J. A. Casas, J. R. Espinosa, A. Ibarra and I. Navarro, Nucl. Phys. B 569 (2000) 82, Nucl. Phys. B 556 (1999) 3, Nucl. Phys. B 573 (2000) 652; S. Antusch, J. Kersten, M. Lindner and M. Ratz, Nucl. Phys. B 674 (2003) 401; S. Antusch, M. Drees, J. Kersten, M. Lindner and M. Ratz, Phys. Lett. B 519 (2001) 238.

[52] L. Alvarez-Gaume, M. Claudson and M. B. Wise, Nucl. Phys. B 207 (1982) 96.

[53] G. F. Giudice and R. Rattazzi, Phys. Rept. 322, 419 (1999)

[54] M. Kawasaki, K. Kohri and T. Moroi, Phys. Rev. D 71 (2005) 083502, Phys. Lett. B 625 (2005) 7

[55] J. R. Ellis, D. V. Nanopoulos and S. Sarkar, Nucl. Phys. B 259 (1985) 175. V. S. Berezinsky, Phys. Lett. B 261 (1991) 71. M. Bolz, W. Buchmuller and M. Plumacher, Phys. Lett. B 443 (1998) 209

[56] W. Buchmuller, P. Di Bari and M. Plumacher, Annals Phys. 315 (2005) 305. G. F. Giudice, A. Notari, M. Raidal, A. Riotto and A. Strumia, Nucl. Phys. B 685, 89 (2004) 